\title{
Three-Dimensional Mid-Infrared Tomographic Imaging of Endogenous and Exogenous Molecules in a Single Intact Cell with Subcellular Resolution**
}

\author{
Luca Quaroni, * Martin Obst,* Marcus Nowak, and Fabio Zobi*
}

\begin{abstract}
Microscopy in the mid-infrared spectral range provides detailed chemical information on a sample at moderate spatial resolution and is being used increasingly in the characterization of biological entities as challenging as single cells. However, a conventional cellular 2D imaging measurement is limited in its ability to associate specific compositional information to subcellular structures because of the interference from the complex topography of the sample. Herein we provide a method and protocols that overcome this challenge in which tilt-series infrared tomography is used with a standard benchtop infrared microscope. This approach gives access to the quantitative $3 D$ distribution of molecular components based on the intrinsic contrast provided by the sample. We demonstrate the method by quantifying the distribution of an exogenous metal carbonyl complex throughout the cell and by reporting changes in its coordination sphere in different locations in the cell.
\end{abstract}

Chemical imaging of cells and tissues relies on the use of spectroscopic techniques sensitive to sample composition in an optical configuration suitable for spectromicroscopy and spectroscopic imaging. Most chemical imaging techniques are based on vibrational spectroscopy or X-ray absorption. ${ }^{[1]}$ Among the vibrational spectroscopy techniques, the absorption of middle infrared (mid-IR) radiation has been particularly successful in providing a detailed description of microscopic molecular composition. It is receiving considerable attention as a microscopy technique to study biochemistry in cells and tissue, both fixed and living, including the uptake and distribution of exogenous compounds. ${ }^{[1,2]}$ In an IR microscopic imaging experiment, the spectroscopic information associated with IR light absorption is coupled to the spatial information contained in the $2 \mathrm{D}$ image formed on

[*] Dr. L. Quaroni, ${ }^{[+]}$Prof. Dr. F. Zobi

Department of Chemistry, University of Fribourg

Chemin de Musée 9, 1700 Fribourg (Switzerland)

E-mail: quaroni@bluewin.ch

fabio.zobi@unifr.ch

Dr. M. Obst, Prof. Dr. M. Nowak

Institute for Geosciences, Eberhard Karls University Tübingen

Hölderlinstrasse 12, 72074 Tübingen (Germany)

E-mail:martin.obst@uni-tuebingen.de

$\left.{ }^{+}\right]$Current address: Functional Genomics Center Zurich Winterthurerstrasse 190, 8057 Zürich (Switzerland)

$[* *$ We are grateful to Holger Marxer, Tübingen, for help with the IR microscope, and to Wolfgang Kürner, Tübingen, for construction of the sample rotation mount.

Supporting information for this article is available a focal plane array (FPA) detector, ${ }^{[3]}$ providing a representation of the chemical composition throughout the sample. ${ }^{[3,4]}$

Despite the wealth of information in a 2D IR image, this format is intrinsically limited because of the difficulty in separating the contribution to the image from the complex topography of the cell. ${ }^{[5]}$ With increased demands being placed on IR imaging experiments, such limitations need to be tackled. The use of attenuated total reflectance (ATR) microscopy techniques can potentially help in some situations where the penetration depth of the evanescent field can be used to gain selective spatial information, but this is not a general case. ${ }^{[6]}$ An important example is provided by efforts to study the cellular uptake of compounds of pharmacological interest, which have been hindered by the difficulty in assessing accurate subcellular distributions from IR images. ${ }^{[7]}$ The study of metabolic processes in cells is another area wherein imaging and accurate quantification of subcellular concentration gradients is of critical importance. ${ }^{[4]}$ However, the possibility of extracting quantitative information from IR absorption spectra relies on the knowledge of the light path through the cellular structure under investigation, which is not available from 2D images. Similar issues are also present in Raman and CARS imaging of cellular systems. However, in contrast to the case of IR absorption measurements, in Raman measurements the shorter depth of focus facilitates $3 \mathrm{D}$ image reconstruction by imaging different planes along the $z$ axis. ${ }^{[7,8]}$ This is not possible with mid-IR radiation, whose depth of focus is frequently greater than cell thickness (see the Supporting Information).

We have addressed these challenges by developing a method to collect three-dimensional IR tomographic images of cellular samples. The general viability of a mid-IR tomography experiment has been proposed by Martin et al. ${ }^{[9]}$ using a synchrotron IR beamline. However, the need for synchrotron light considerably restricts the applicability of the method. We now show that the chemical imaging capability of mid-IR tomography for subcellular imaging can be fully implemented with a benchtop microscope and a conventional light source.

We measured tilt-series transmission images of a single layer of cells from the epidermis of Allium cepa. Experimental details are reported in the Supporting Information (SI). The experimental setup is shown in Figure SI1. The cells are brick-shaped and are about $180 \mu \mathrm{m} \times 80 \mu \mathrm{m} \times 40 \mu \mathrm{m}$ in size. Figure 1 shows 2D images of a single cell using absorption contrast from selected bands in the IR spectrum and the corresponding $3 \mathrm{D}$ reconstructions. The $2 \mathrm{D}$ images are IR pixel maps where the gray scale corresponds to the absorbance of selected bands, with white being the highest absorb- 

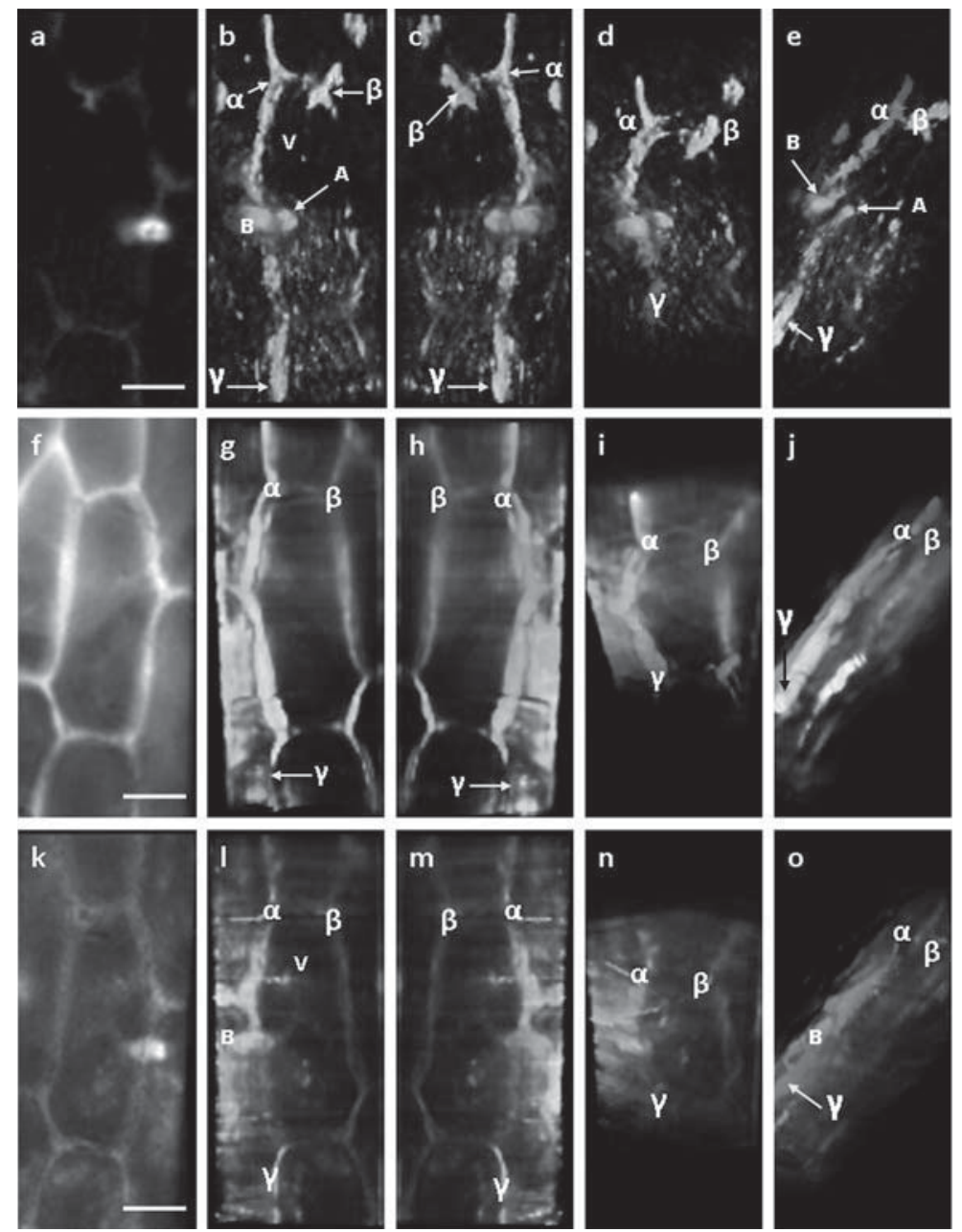

Figure 1. 3D tomographic IR images of Allium cepa cells with absorption contrast from molecular components. a) 2D IR absorption image at $1538 \mathrm{~cm}^{-1}$. b-e) Views at different tilt angles used for the $3 \mathrm{D}$ reconstruction at $1740 \mathrm{~cm}^{-1}$. f) $2 \mathrm{D}$ IR absorption image at $1740 \mathrm{~cm}^{-1} \cdot g-j$ ) Views at different tilt angles used for the 3D reconstruction at $1740 \mathrm{~cm}^{-1}$. k) 2D IR absorption image at $2917 \mathrm{~cm}^{-1}$. I-0) Views at different tilt angles used for the 3D reconstruction at $2917 \mathrm{~cm}^{-1} . \alpha, \beta, \gamma$ show the location of three points on the cell wall and serve as a visual aid to assess the orientation of the cell in the images. $A$ and $B$ are used to label the structures discussed in the text. $V$ indicates the location of a vacuole. Scale bar: $50 \mu \mathrm{m}$.
One notable case in this particular sample is the identity and location of cell nuclei. The visible photographs and 2D IR images of Figure 1 show the presence of several structures within the cells in the size range of nuclei. In particular, two of these structures, marked $\mathbf{A}$ and $\mathbf{B}$ in Figure 1 b,e, overlap. A difference in the composition of the two structures is apparent, based on the different intensities of the bands at $1538 \mathrm{~cm}^{-1}$ (Figure $1 \mathrm{~b}$ e) and at $2917 \mathrm{~cm}^{-1}$ (Figure $1 \mathrm{k}-\mathrm{o}$ ). In $\mathbf{A}$ absorption from alkyl chains at $2917 \mathrm{~cm}^{-1}$ is much lower absorption than in $\mathbf{B}$, indicating lower lipid content. In 2D IR images, this difference in spectral absorption does not disprove the assignment of either structure to the nucleus, since the adsorption could arise from the contribution of other cellular components above or below the structure. In contrast, 3D images are unambiguous. Figure 2 shows frames extracted from one reconstruction that highlight the detailed structure of the cellular components of interest. Only structure $\mathbf{A}$ is located in the same plane as the cell wall. Structure $\mathbf{B}$ is located on a plane outside the cell plane and extends over the cell wall, therefore excluding its identification with a nucleus. In addition, structure A is barely visible in Figure $1 \mathrm{k}-\mathrm{o}$, indicating low lipid content, as expected for a nucleus. ${ }^{[5]}$ Structure B appears to be a droplet with a high content of long-chain alkanes, probably a spherosome (lipid body). Discriminating between spherosomes and nuclei is normally possible in optical microscopy by selective staining. ${ }^{[10]}$ In contrast, in our experiment we show that these cell structures can be identified without staining.

Several structures that extend from the cell wall, such as filaments that are 5-7 $\mu \mathrm{m}$ in diameter, can be visualized also at $2917 \mathrm{~cm}^{-1}(\lambda=3.4 \mu \mathrm{m})$, indicating that the spatial resolution of the image is good. Similar conclusions can be drawn by observing the separation between the nucleus and the spherosome in Figure 1 and in Figure 2. At $1538 \mathrm{~cm}^{-1}(\lambda=6.5 \mu \mathrm{m})$ the nucleus, $20-25 \mu \mathrm{m}$ in size, is clearly defined and resolved from the nearby ance. A sample spectrum, highlighting specific absorption bands is shown in the Supporting Information (Figure SI2).

As examples we show 3D images of three selected bands from cellular components. The absorption at $1538 \mathrm{~cm}^{-1}$ (Figure 1a-e) is characteristic of the amide group of polypeptides and is often stronger in the location of the nucleus. The absorption at $1740 \mathrm{~cm}^{-1}$ (Figure $1 \mathrm{f}-\mathrm{j}$ ) is attributed primarily to the carbonyl groups of esters. Acetylated polysaccharides are the main contributors in the plant cell wall. The absorption at $2917 \mathrm{~cm}^{-1}$ (Figure $1 \mathrm{k}-\mathrm{o}$ ) is characteristic of the alkyl chains of fatty acids. Tomographic images constructed from these absorption bands clearly render the spatial distribution of subcellular structures, including the cell wall, the nucleus, and a large vacuole, marked with $\mathrm{V}$ in Figure 1, which occupies much of the cellular volume. spherosome, in contrast to what is observed in 2D IR images, where the two structures appear to overlap. Figure SI 4 shows that the two objects, $8.5 \mu \mathrm{m}$ apart, are fully resolved according to Rayleigh's criterion. The resolution shown in 3D images is comparable to that obtained with $2 \mathrm{D}$ images using the same instrument. Although we did not quantitatively assess the resolution of this imaging configuration, and we expect it to be somewhat inferior to the diffraction-limited resolution, the possibility of resolving objects as little as $8.5 \mu \mathrm{m}$ apart suggests that that it is close to diffraction-limited values ( $\approx 5 \mu \mathrm{m}$ at $2917 \mathrm{~cm}^{-1}$ and $\approx 10 \mu \mathrm{m}$ at $1538 \mathrm{~cm}^{-1}$ with the optics used in this measurement; see the Supporting Information). the subcellular localization of an exogenous compound by mapping the $3 \mathrm{D}$ distribution of an octahedral trisaqua tris-
We tested the possibility of using IR tomography to study 

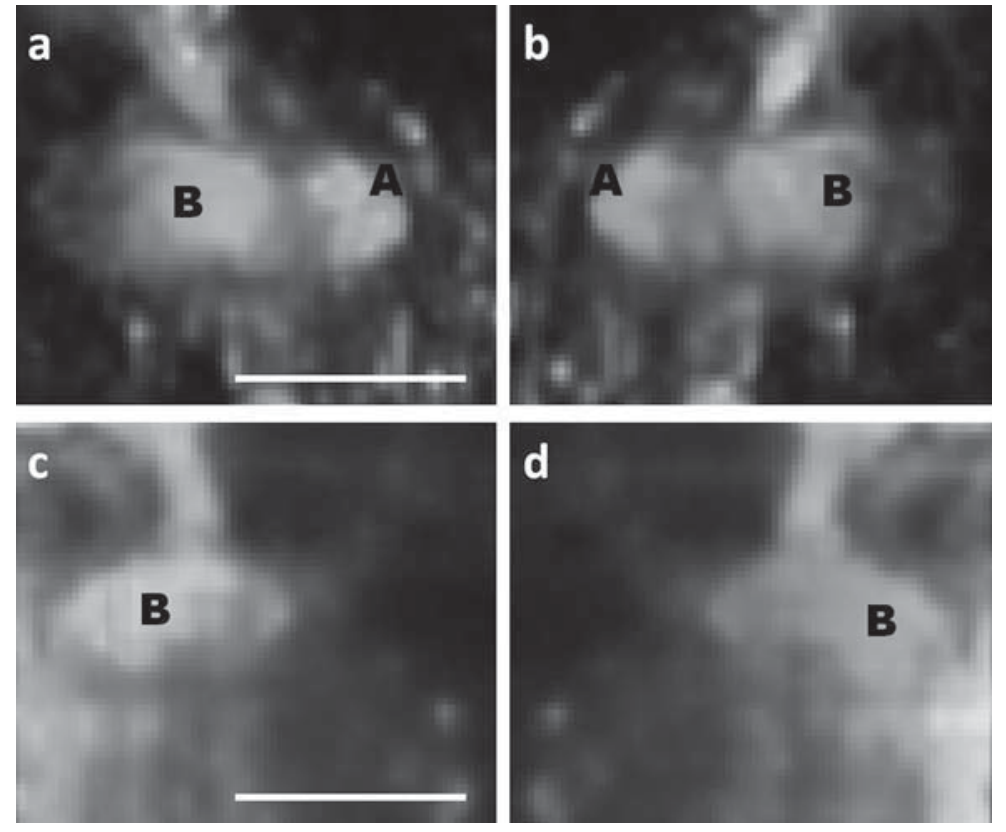

Figure 2. Detail of the nucleus of Allium cepa cells. a,b) 3D reconstruction at $1538 \mathrm{~cm}^{-1}$ viewed at different angles. c,d) $3 D$ reconstruction at $2917 \mathrm{~cm}^{-1}$ viewed at different angles. A and B are used to label the particles discussed in the text. Scale bar: $50 \mu \mathrm{m}$.

carbonyl rhenium complex $\left[\operatorname{Re}(\mathrm{CO})_{3}\left(\mathrm{H}_{2} \mathrm{O}\right)_{3}\right]^{+}(\mathbf{1}$, see Scheme SI 1 for structural details). Compound $\mathbf{1}$ is widely employed as a synthon for the generation of CO-releasing molecules, a new class of pharmaceutical compounds currently under evaluation. ${ }^{[11]}$

The viability of $2 \mathrm{D}$ mid-IR imaging of the cellular uptake of metal carbonyl complexes has already been demonstrated. ${ }^{[7,12]}$ Compound $\mathbf{1}$ shows two intense $\mathrm{CO}$ bands (A1 and E based on their symmetry properties) in the 2050$1850 \mathrm{~cm}^{-1}$ region of the vibrational spectrum (Figure SI2). We measured the uptake of $\mathbf{1}$ using energy-dispersive X-ray microanalysis (EDXMA) (Figure SI3) and reconstructed its distribution using the $\mathrm{CO}$ absorption bands (A1 at $2021 \mathrm{~cm}^{-1}$ and $\mathrm{E}$ at $1890 \mathrm{~cm}^{-1}$ ). The tomographic image of the A1 stretching band indicates preferential accumulation of the molecule within the cell wall and the nucleus (Figure 3). It is notable that the accumulation of $\mathbf{1}$ within the lipid body is negligible, a feature that would be difficult to appreciate in $2 \mathrm{D}$ images. In previous 2D IR imaging and mapping experiments addressing the cellular distribution of compounds related to $\mathbf{1}$ there were difficulties in interinterpreting IR maps in the proximity of the nucleus because of interfering cellular topography. ${ }^{[7]}$ In contrast, in Figure 3 the partitioning of $\mathbf{1}$ between the nucleus and other cellular components is unequivocal. For additional detail, a 3D rotating reconstruction of the A1 distrubution is provided in the Supporting Information.

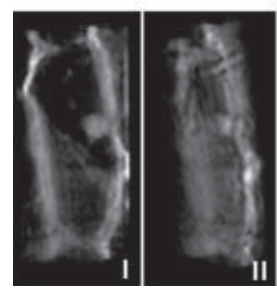

The concentration of $\mathbf{1}$ can be quantified in various locations of the cell by using the BeerLambert law (see the Supporting Information) on the 2D IR image of band A1 in Figure 3. An accurate value of the optical path in various locations can be obtained from the $3 \mathrm{D}$ reconstructions. As an example, the concentration of $\mathbf{1}$ in the cell nucleus may be quantified at $10 \mathrm{~mm}$.

The availability of reconstructed 3D distributions of spectral bands allows the quantitative analysis of the colocalization of endogenous and exogenous chemical species by various forms of correlation analysis. Figure 4 shows an example of such an approach which is based on a $2 \mathrm{D}$ histogram in which the 3D distributions of two absorption bands are plotted against each other, on a voxel by voxel basis; this is frequently referred to as a scatterplot. ${ }^{[13]}$ Bands of species that are highly correlated in space give plots with a distribution of maxima along a slope. In contrast, bands that are not correlated in space give plots with a different trend or without any obvious trend. Bands from the same molecule show identical distribution and a narrow diagonal band in the scatter plot
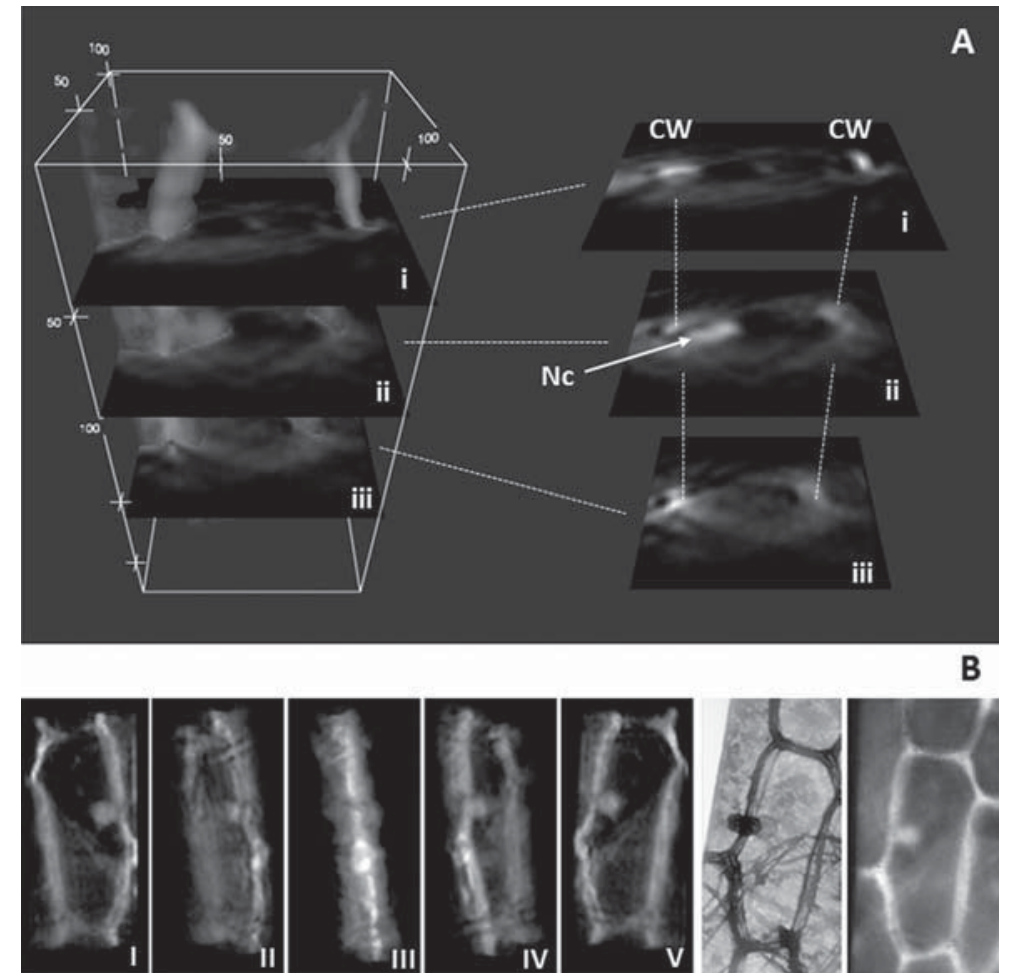

Figure 3. 3D computed tomography reconstruction of the cellular distribution of the A1 stretching frequency of 1 . A) Reconstructed volume rendering with digital slicing at selected locations. Stacked slices on the right correspond to volume positions on the left. $\mathrm{CW}=$ cell wall; $\mathrm{Nc}=$ nucleus. Vertical dotted lines connect locations of highest $\mathrm{A} 1$ area intensity on the $\mathrm{CW}$. B) Volume rendering visualized as a function of rotation about the vertical axis. From left to right: $45^{\circ}$ rotation steps starting at $0^{\circ}(\mathrm{I})$ to $180^{\circ}(\mathrm{V})$. The rotation series is accompanied by a laser scanning transmission image at $488 \mathrm{~nm}$ illustrating a projection of the detailed structure of the cell and one of the 2D IR maps of the Al band used for reconstruction. 


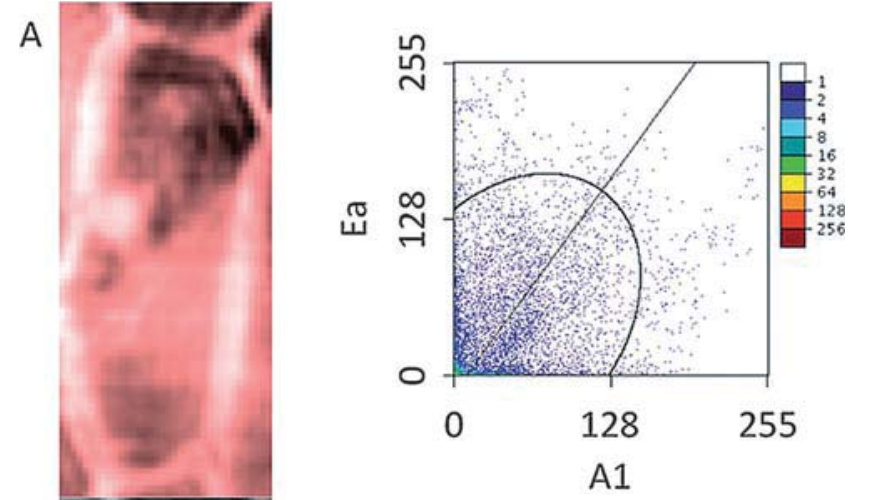

B

C

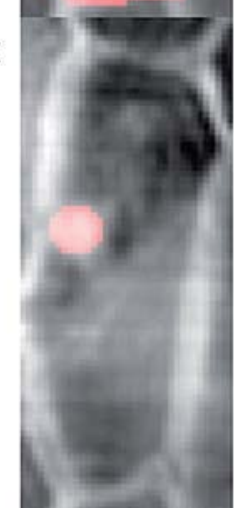

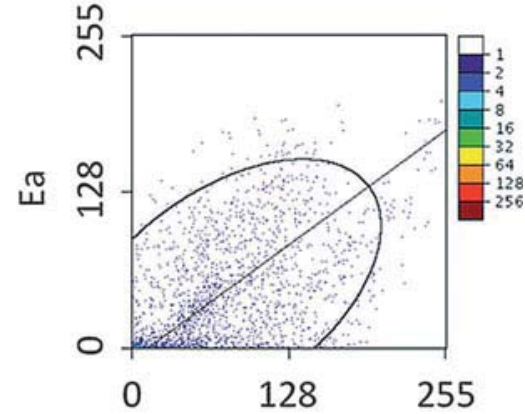

A1

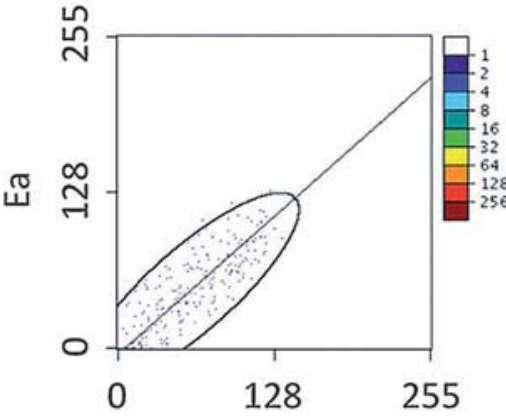

A1

As an example, Figure 4 shows the scatter plots of the A1 and Ea bands of complex $\mathbf{1}$. The plots are calculated over the whole cell (Figure 4A) and over restricted volumes of the sample, such as the cell wall (Figure 4B) and the nucleus (Figure 4C). It is immediately evident that the scatterplot for the nucleus shows high correlation between band A1 at $2021 \mathrm{~cm}^{-1}$ and the component of band $\mathrm{E}$ at $1881 \mathrm{~cm}^{-1}$ (named Ea), while the other plots show a much poorer correlation. The bands from the nucleus must arise from one specific molecular species. In other cellular locations, complex 1 shows a heterogeneous structure, giving rise to multiple $\mathrm{A} 1$ and $\mathrm{E}$ bands and thus lower correlation. The observation suggests that uptake of $\mathbf{1}$ within the nucleus has occurred according to a specific mechanism that ensured structural homogeneity of the coordination sphere. In contrast, interaction with the cell wall is likely due to nonspecific binding of the rhenium center to the polysaccharide frame and gives rise to a heterogeneous coordination sphere.

The introduction of a protocol for $3 \mathrm{D}$ tomographic imaging in the mid-IR region provides a tool to greatly expand the capabilities of mid-IR microscopy in cellular physiology. In our work we show that experiments on cells can be easily performed by using a conventional benchtop machine with an FPA detector and a thermal source. The possibility of performing mid-IR tomography on a benchtop microscope is anticipated to have major implications on the impact of the technique. Benchtop IR microscopes can be easily set up in any cell biology laboratory. We expect that the introduction of cellular IR tomography will be one of the breakthroughs that will revolutionize IR imaging of biological systems, together with the development of near-field imaging with sub-wavelength resolution. ${ }^{[15]}$ The applicability of the technique is general and not limited to plant cells. The same protocols described in this work can be used for benchtop tomographic imaging of any cells and any other samples accessible by an IR transmission measurement.

Figure 4. 2D histograms of bands $A 1$ and $E a$ in different subvolumes of the reconstructed cell. A) Scatterplot of the whole cell volume. The distribution is widespread and the appearance of clusters of data points indicates that there regions in which $\mathrm{A} 1$ and Ea correlate as well as regions in which the components appear to be independent. The line shows the linear regression line calculated as the major axis and the $95 \%$ confidence ellipse of this linear model to describe the data. B) Scatterplot of the region of the cell wall. When the analysis was restricted to the cell wall, the data scatter more closely to the major axis, as indicated by the elongated confidence ellipse. C) In the region of the cell nucleus, the two species $\mathrm{Ea}$ and $\mathrm{A} 1$ are highly correlated as shown by the narrow confidence ellipse. The root mean square errors (RMSE) of the fits are reduced from 38 (A) to 34 (B) to $14(\mathrm{C})$.

(Figure 4). Quantifying the correlation of band distributions is a rapid way to identify spectral features that are colocalized in the cell. It also makes it possible to identify bands from the same molecule, which must be highly correlated. The latter approach is a very important tool in resolving the complexity of cellular IR spectra. ${ }^{[14]}$

Keywords: chemical imaging - infrared tomography . single-cell studies

[1] a) M. Obst, G. Schmid, Methods Mol. Biol. 2014, 1117, 757-781; b) A. F. Palonpon, J. Ando, H. Yamakoshi, K. Dodo, M. Sodeoka, S. Kawata, K. Fujita, Nat. Protoc. 2013, 8, 677-692; c) A. F. Palonpon, M. Sodeoka, K. Fujita, Curr. Opin. Chem. Biol. 2013, 17, 708-715; d) J. P. Pezacki, J. A. Blake, D. C. Danielson, D. C. Kennedy, R. K. Lyn, R. Singaravelu, Nat. Chem. Biol. 2011, 7, 137-145; e) L. Quaroni, T. Zlateva, Analyst 2011, 136, 3219-3232; f) G. Schmid, F. Zeitvogel, L. Hao, P. Ingino, M. Floetenmeyer, Y. D. Stierhof, B. Schroeppel, C. J. Burkhardt, A. Kappler, M. Obst, Geobiology 2014, 12, 340-361; g) G. Schmid, F. Zeitvogel, L. Hao, P. Ingino, W. Kuerner, J. J. Dynes, C. Karunakaran, J. Wang, Y. Lu, T. Ayers, C. Schietinger, A. P. Hitchcock, M. Obst, Microsc. Microanal. 2014, 20, 531536; h) J. Wang, A. P. Hitchcock, C. Karunakaran, A. Prange, B. Franz, T. Harkness, Y. Lu, M. Obst, J. Hormes, AIP Conf. Proc. 2011, 1365, 215-218. 
[2] A. Kretlow, Q. Wang, J. Kneipp, P. Lasch, M. Beekes, L. Miller, D. Naumann, Biochim. Biophys. Acta Biomembr. 2006, 1758, 948-959.

[3] I. W. Levin, R. Bhargava, Annu. Rev. Phys. Chem. 2005, 56, $429-$ 474.

[4] a) P. Lasch, D. Naumann, Biochim. Biophys. Acta Biomembr. 2006, 1758, 814-829; b) C. Matthäus, B. Bird, M. Miljkovic, T. Chernenko, M. Romeo, M. Diem, Methods Cell Biol. 2008, 89, 275-308; c) L. Quaroni, T. Zlateva, B. Sarafimov, H. W. Kreuzer, K. Wehbe, E. L. Hegg, G. Cinque, Biophys. Chem. 2014, $189,40-48$.

[5] P. Lasch, A. Pacifico, M. Diem, Biopolymers 2002, 67, 335-338.

[6] a) M. K. Kuimova, K. L. Chan, S. G. Kazarian, Appl. Spectrosc. 2009, 63, 164-171; b) T. P. Wrobel, K. M. Marzec, K. Majzner, K. Kochan, M. Bartus, S. Chlopicki, M. Baranska, Analyst 2012, 137, $4135-4139$.

[7] a) S. Clède, F. Lambert, C. Sandt, Z. Gueroui, N. Delsuc, P. Dumas, A. Vessières, C. Policar, Biotechnol. Adv. 2013, 31, 393 395; b) F. Zobi, L. Quaroni, G. Santoro, T. Zlateva, O. Blacque, B. Sarafimov, M. C. Schaub, A. Y. Bogdanova, J. Med. Chem. 2013, 56, 6719-6731.

[8] B. R. Wood, P. R. Stoddart, D. McNaughton, Aust. J. Chem. 2011, 64, 593-599.
[9] M. C. Martin, C. Dabat-Blondeau, M. Unger, J. Sedlmair, D. Y. Parkinson, H. A. Bechtel, B. Illman, J. M. Castro, M. Keiluweit, D. Buschke, B. Ogle, M. J. Nasse, C. J. Hirschmugl, Nat. Methods 2013, 10, 861-864.

[10] G. E. Holcomb, A. C. Hildebrandt, R. F. Evert, Am. J. Bot. 1967, $54,1204-1209$.

[11] a) R. Motterlini, L. E. Otterbein, Nat. Rev. Drug Discovery 2010 , 9, 728 -U724; b) F. Zobi, Future Med. Chem. 2013, 5, 175-188.

[12] S. Clède, F. Lambert, C. Sandt, S. Kascakova, M. Unger, E. Harte, M. A. Plamont, R. Saint-Fort, A. Deniset-Besseau, Z. Gueroui, C. Hirschmugl, S. Lecomte, A. Dazzi, A. Vessières, C. Policar, Analyst 2013, 138, 5627-5638.

[13] F. Zeitvogel, G. Schmid, L. Hao, P. Ingino, M. Obst, J. Microsc., DOI: $10.1111 /$ jmi.12187.

[14] L. Quaroni, T. Zlateva, Anal. Chem. 2014, 86, 6887-6895.

[15] a) I. Amenabar, S. Poly, W. Nuansing, E. H. Hubrich, A. A. Govyadinov, F. Huth, R. Krutokhvostov, L. Zhang, M. Knez, J. Heberle, A. M. Bittner, R. Hillenbrand, Nat. Commun. 2013, 4, 2890; b) H. A. Bechtel, E. A. Muller, R. L. Olmon, M. C. Martin, M. B. Raschke, Proc. Natl. Acad. Sci. USA 2014, 111, $7191-7196$. 